\title{
Low-cost piezoelectric footswitch system for measuring temporal parameters during walking
}

\author{
Gustavo Balbinot ${ }^{1 *}$, Clarissa Pedrini Schuch ${ }^{2}$, Milton Antonio Zaro ${ }^{3}$, Marco Aurélio Vaz ${ }^{2}$ \\ ${ }^{1}$ Department of Neuroscience, Federal University of Rio Grande do Norte (UFRN), Brain Institute, Natal, RN, Brazil \\ ${ }^{2}$ School of Physical Education, Federal University of Rio Grande do Sul (UFRGS), Porto Alegre, RS, Brazil \\ ${ }^{3}$ Biomechanics Lab., Brazilian Institute for Shoes, Leather and Parts (IBTeC), Novo Hamburgo, RS, Brazil \\ *Corresponding author E-mail: gustavo.balbinot@hotmail.com
}

Copyright (c) 2014 Gustavo Balbinot et. al. This is an open access article distributed under the Creative Commons Attribution License, which permits unrestricted use, distribution, and reproduction in any medium, provided the original work is properly cited.

\begin{abstract}
Human walking is one of the most investigated biomechanical events, and gait analysis depends on accurate measurement of heel strike (HS) and toe off (TO). The purpose of this study was to construct and validate a low-cost footswitch system for the measurement of temporal gait parameters. Ten young healthy subjects participated of the validation and test of the footswitch system with two different footwear, Bland-Altman analysis showed $98 \%$ and $95 \%$ of validation data within the limits of agreement, for HS and TO respectively (mean difference of $16 \mathrm{~ms} \pm 1 \mathrm{~ms}$ and $20 \mathrm{~ms} \pm 9 \mathrm{~ms}$ ) and the temporal parameters measured during treadmill walking at a speed of $4.5 \mathrm{~km} . \mathrm{h}^{-1}$ showed results similar to those found in the literature for normal walking. The outcomes confirm low CoVs for the instrumented athletic and instability shoe, respectively: $(1.52 \pm 0.61) \%$ and $(1.90 \pm 0.73) \%$ for contact time, $(2.17 \pm 0.95) \%$ and $(2.57 \pm 0.95) \%$ for balance time, $(0.84 \pm 0.28) \%$ and $(1.12 \pm 0.53) \%$ for stride time. The low-cost footswitch system described and validated in the present study has an important practical applicability, mostly for emerging and developing countries biomechanics labs.
\end{abstract}

Keywords: Footswitch System, Gait Analysis, Locomotion, Low-Cost, Walk.

\section{Introduction}

Gait analysis is one of the most studied phenomena in the field of biomechanics, and relies upon precise determination of gait events, such as heel strike (HS) and toe off (TO)[1,2]. This information is necessary to calculate several gait biomechanical parameters (e.g., step frequency, percentage of single and double support phases) [3]. Also, it is useful to normalize and synchronize other devices such as those that collect neuromuscular activation data during the gait cycle or to synchronize functional electrical stimulation (FES) devices, both in normal and pathological gait [4].

In clinical practice a simple method to quantify gait asymmetry or abnormality is necessary when treating musculoskeletal injury, pathology, or balance disorders [5]. Force plates are the gold standard for determining HS and TO during gait $[2,6,7]$. Despite their accuracy, force plates are a relatively expensive item of equipment, especially for biomechanics laboratories located in economically developing countries. High equipment costs are a reason to seek alternative methods to quantify temporal parameters in gait analysis [8].

In these cases, other types of sensors can be used to quantify HS and TO during walking, including accelerometers and footswitch systems. The latter have been used to measure gait temporal parameters for decades $[6,7,9,10]$. In addition, they are widely used for rehabilitation during assisted gait and for FES synchronization during walking [11], and to synchronize the activity of exoskeletons [12]. Compared to a footswitch system, accelerometers have a lower accuracy (mean absolute error of $2.4 \mathrm{~ms} \pm 2.1 \mathrm{~ms}$ for the footswitch system compared to $9.5 \mathrm{~ms} \pm 9.0 \mathrm{~ms}$ for the accelerometer system) [13]. Furthermore, determining temporal parameters using accelerometers, despite their portability, requires the use of correction factors and intense mathematical processing through specific computational algorithms, a fact that complicates the use of real time results $[14,15]$. 
Video analysis systems can also be used to determine temporal gait parameters [2]. However, determining of HS and TO can be problematic with low-speed cameras, i.e., low sampling rate (below $50 \mathrm{~Hz}$ for walking) [7]. For example, in a low-cost kinematic system with a sampling of 60 samples per second, the maximal resolution is \pm 17 ms [7]. Usually low-cost cameras, such as web cams, have low sampling rates. Furthermore, obtaining kinematic data using low cost camera systems is usually not an automated process, which presents a subjective factor and prevents the use of information in real time (e.g., for FES) [12]. Considering these facts, the purpose of this study was to construct and validate a low-cost footswitch system $(<10$ USD) to measure the timing of critical events during the gait cycle, this system serves as a basis to calculate temporal parameters during normal gait and to synchronize other devices during gait analysis. A secondary goal was to verify the practical applicability of the footswitch system during treadmill walking.

\section{Methods}

\subsection{Construction of the system}

For the instrumented insole construction two piezoelectric sensors (see supplementary material for sensor description) were attached on the underside of a rubber EVA sheet insole (4 mm thick). In the present study the piezoelectric sensor was obtained from a buzzer or beeper, which is an audio signaling device, which may be mechanical, electromechanical, or piezoelectric; the external plastic cape was removed, and only the piezoelectric sensor was utilized with no power supply or amplifying requirements. Circular cuts matching the shape of the piezoelectric sensors were made $1 \mathrm{~mm}$ deep, using a utility knife. Cuts were located in the insole region corresponding to the hallux and heel regions of the plantar surface of the foot. The sensors were fixed to the EVA insole circular cuts with silicone glue, which was applied only to the external metallic circle of the piezoelectric sensor (Fig. 1b).

The response of the piezoelectric sensors during walking was measured using a digital oscilloscope to set the system up (DS1102C, $100 \mathrm{MHz}$, Rigol, Ohio, USA). Once a voltage response above A/D converter capacity was evidenced (i.e., exceeding $10 \mathrm{~V})$, an electric resistance was utilized in parallel for each sensor $(\approx 33 \Omega$, Fig. 1$)$, thus the voltage did not exceed a maximum of about $\pm 2 \mathrm{~V}$.

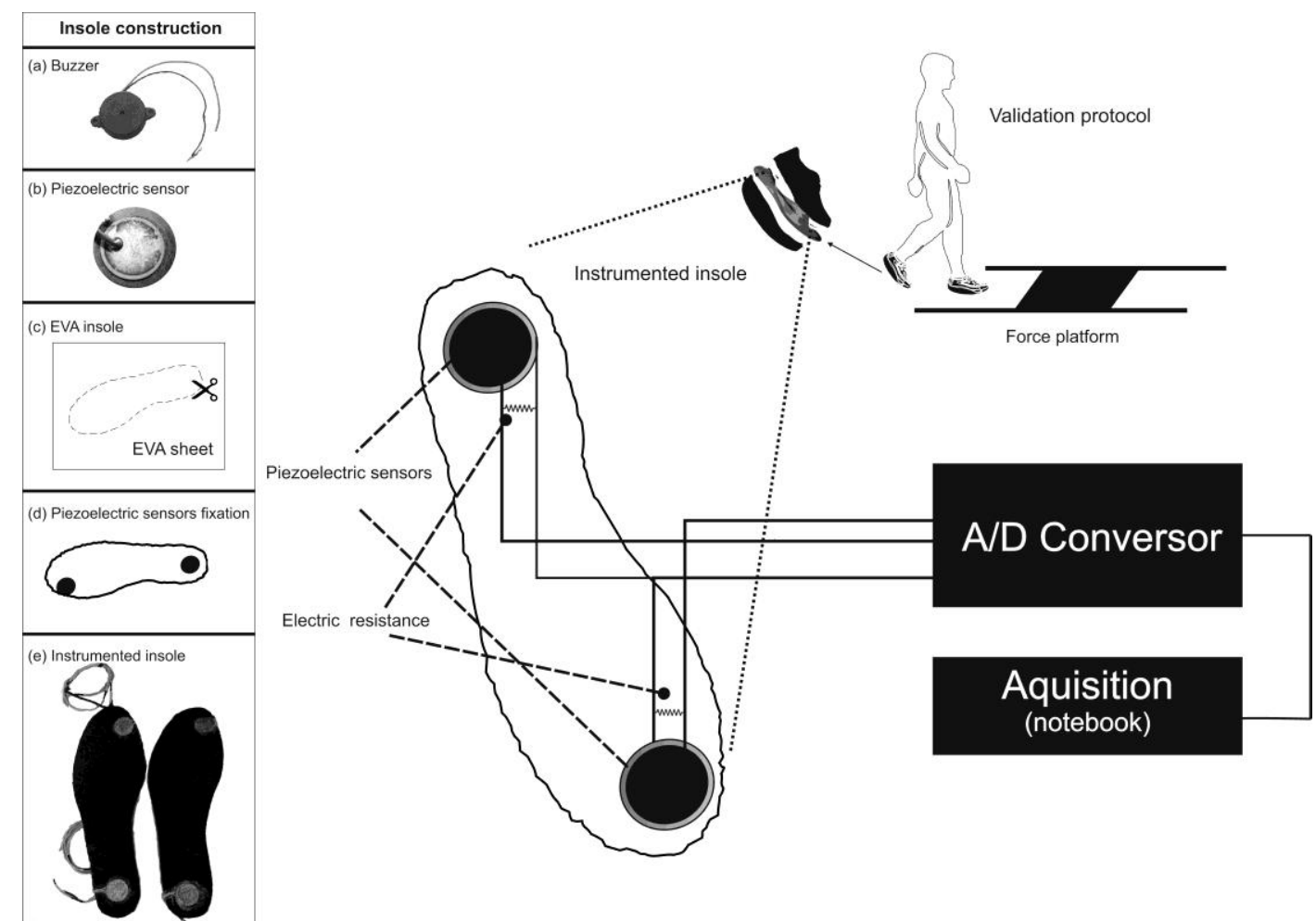

Fig. 1: Instrumented insole outlook and construction (left panel), connection diagram and validation protocol (right). Left panel: (a) Piezoelectric buzzer used in alarm devices, the plastic cap is removed and the piezoelectric sensor is exposed; (b) Piezoelectric sensor after the plastic cap removal; (c) EVA insole construction (4 mm EVA sheet); (d) Circular cuts are made in the same shape of the piezoelectric sensors (1 mm deep) and these sensors are fixed with silicon glue applied to the outer border (see b) and (e) footswitch system. Piezoelectric sensors voltage responses were reduced by electrical resistances $(\approx 33 \Omega$ ) positioned in parallel and connected to the A/D converter. The validation of the footswitch system (upper right) consists of a one-step protocol with synchronous data acquisition from the footswitch system and the vertical component of the ground reaction force $\left(\mathrm{GRF}_{\mathrm{V}}\right)$. 


\subsection{Experimental protocol}

Ten subjects (4 male: $29.1 \pm 5.9$ years, BM $82.9 \pm 7.9 \mathrm{~kg}$; 6 female: $28.7 \pm 2.1$ years, BM $57.3 \pm 8.2 \mathrm{~kg}$ ) participated of the present study. The study was approved by the ethics committee on research at the Federal University of Rio Grande do Sul, and all participants signed a consent form. The participants were recruited intentionally and were research colleagues at Federal University of Rio Grande do Sul, the sample size was calculated based in previous studies of our research group.

\subsection{Validation of the system}

For the footswitch system validation a force plate (OR-6, FN $\approx 530$, Advanced Mechanical Technology Inc., Massachusetts, USA) was used simultaneously with the footswitch system, which consisted of an A/D converter (NI623, National Instruments, USA) and the instrumented insole placed inside an athletic shoe. Footswitch system validation was performed by means of the force plate system, considered the gold standard for measuring temporal parameters [2]. The participants performed ten steps each on the force plate. The one step protocol was used, at which subjects performed a step with the right foot on the force plate and at least two more steps after the force plate step, subjects were asked to perform different walking speeds in a random order (the speed was not controlled) (Fig. 1) [16]. Subjects walked on the force plate wearing the instrumented insole inserted inside the footwear; two distinct types of footwear were utilized (i.e. athletic shoes and instability shoes). To ensure the gold standard measure by means of the force plate, previously to each data collection the hardware zero button was pressed and the HS and TO was defined as when the force signal exceeds or fall from a $5 \mathrm{~N}$ threshold, respectively. Vertical ground reaction force $\left(\mathrm{GRF}_{\mathrm{V}}\right)$ and footswitch system data were acquired simultaneously in a custom-written LabVIEW ${ }^{\circledR}$ software data acquisition and processing routine at $2000 \mathrm{~Hz}$ (Fig. 2).

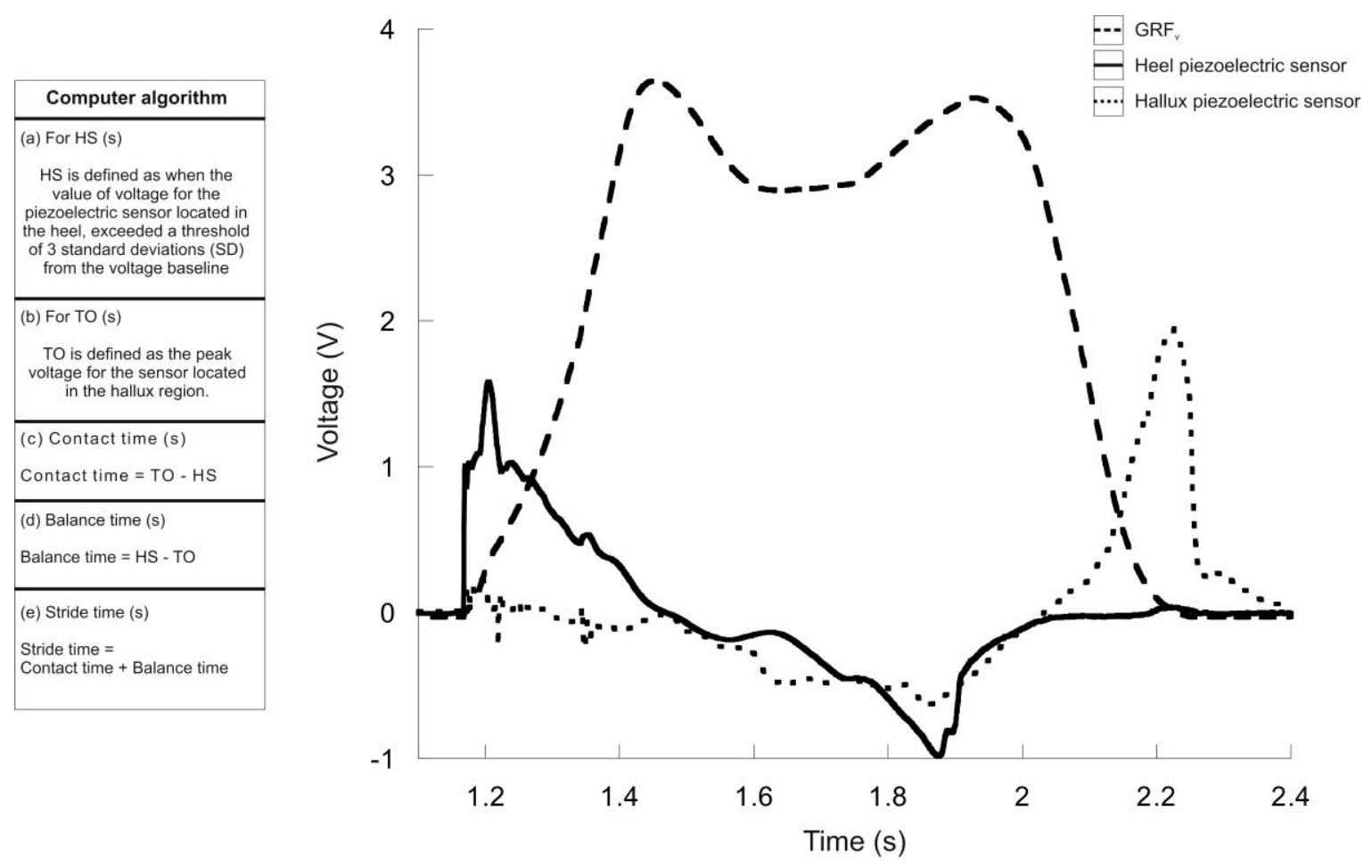

Fig. 2: Representative data from one subject of the validation protocol measurement. Broken line is the vertical component of the ground reaction force $\left(G R F_{V}\right)$, dotted line is the hallux piezoelectric sensor voltage response and full line is the heel piezoelectric sensor voltage response. HS was defined when the value of voltage for the piezoelectric sensor located in the heel, exceeded a threshold of 3 standard deviations (SD) from the voltage baseline value. TO was defined as the peak voltage for the sensor located in the hallux region; the characterization of TO was based in a visual qualitative analysis during pilot studies and was confirmed during the footswitch system validation.

\subsection{Reliability of the system}

After protocol validation the footswitch system was tested during treadmill walking (Inbramed Master, $2070 \mathrm{~mm} \times 770$ $\mathrm{mm}, 250 \mathrm{~kg}, 0-16 \mathrm{~km} \cdot \mathrm{h}^{-1}$, Porto Alegre, Brazil). For the assessment of the reliability of the footswitch system, data from twenty step cycles were collected per subject (ten for each footwear type: athletic shoes and instability shoes). Different types of footwear were used in order to test the footswitch system responses to different conditions, once the instrumented insole is positioned inside the footwear. The temporal parameter analyzed were: contact time (i.e., period 
of time in seconds at which the foot contacts the ground), balance time (i.e., period of time in seconds at which the foot do not make contact with the ground and is conducted to the next ground contact) and stride time (i.e., period of time in seconds of contact time summed with the balance time). These temporal parameters were measured online during treadmill walking at a speed close to the self selected speed (i.e., $4.5 \mathrm{~km} . \mathrm{h}^{-1}$ ) using the low-cost footswitch system and the temporal parameters $\mathrm{CoV}$ was calculated afterward in an offline procedure.

\subsection{Data acquisition and processing}

Data acquisition and processing were performed by means of LabVIEW ${ }^{\circledR} 8.5$ platform through DATAQ function, where the two piezoelectric sensors were synchronized with the $\mathrm{GRF}_{\mathrm{V}}$ at a sampling rate of $2000 \mathrm{~Hz}$. For data processing a mathematical routine was developed in order to: (1) filter the data (low-pass butterworth filter, order 4, 10

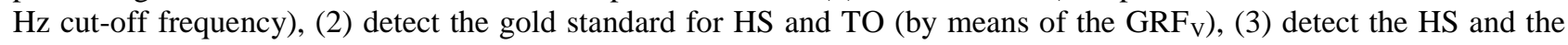
TO for piezoelectric sensors, (4) calculate the difference in contact time between the two methods, i.e., force plate and footswitch system.

In this footswitch system, HS and TO characterization depends on the piezoelectric sensors voltage response to the applied force and walking mechanics. According to the physical properties of piezoelectric sensors, this sensor can be modeled as a voltage source with high impedance followed by a high-pass filter. The source of this tension is directly proportional to the applied pressure on the sensor. The output signal is then the voltage source after passing through the filter of the equivalent circuit, which has its own characteristics for each sensor.

From this physical property of piezoelectricity and the dynamics of gait, the characterization of HS was defined when the value of voltage for the piezoelectric sensor located in the heel, exceeded a threshold of 3 standard deviations (SD) from the voltage baseline value, according to previous knowledge of our research group. TO was defined as the peak voltage for the sensor located in the hallux region, since in the moment which the foot is removed from the ground, the voltage response is maximum and only reaches zero during the swing phase; once the energy of the system must return to zero after the applied force, TO was defined as the peak voltage signal in the hallux sensor (Fig. 2). The characterization of TO was based in a visual qualitative analysis during pilot studies and was confirmed during the footswitch system validation (Bland-Altman analysis); and it is direct related to the piezoelectric sensor dynamic response when compared to the force plate signal.

\subsection{Statistical analysis}

Descriptive statistics with mean and SD was used. For the footswitch system validation Bland and Altman's limits of agreement analysis was accomplished to verify footswitch system data agreement to the gold standard [17]. Furthermore a histogram was plotted to qualitatively verify the statistical distribution of the validation data. For the assessment of the reliability of the footswitch system, the $\mathrm{CoV}$ was calculated as $\mathrm{CoV}=[(\mathrm{SD} / \mathrm{mean}) \mathrm{x} 100]$, for each subject. The $\mathrm{CoV}$ mean and SD were used to analyze the temporal parameters variability during walking with the two types of footwear previously described [18]. The temporal parameters analyzed were: contact time, balance time and stride time.

\section{Results}

The assessment of the between methods agreement was $98 \%$ for HS and 95\% for TO (Fig 3). The relationship between HS and TO events detection by the footswitch system and the gold standard showed a good agreement, with only a few plots outside the limits of agreement.

Fig. 4 shows the temporal differences between HS and TO events detection, of $-16 \mathrm{~ms}$ (28\% of cases) and $-20 \mathrm{~ms}(27 \%$ of cases) respectively. The negative sign indicates that the footswitch system has detected, both HS and TO, with delay when compared to the force plate. Furthermore, the average difference between methods was (16 \pm 1$) \mathrm{ms}$ and $(20 \pm 9) \mathrm{ms}$ for HS and TO, respectively.

Table 1 describes the footswitch system based temporal measurements during treadmill walking at $4.5 \mathrm{~km} . \mathrm{h}^{-1}$ with different types of footwear, data magnitude corroborate with previous studies for this speed. Furthermore, data variability (i.e., SD and $\mathrm{CoV}$ ) were similar for both footswitch system's equipped footwear and, showed low SD and $\mathrm{CoV}$ for all variables. Although the $\mathrm{CoV}$ for the treadmill walking include variability due to the transducer system, as well as variability in loading during gait, this measurement provide a good parameter for comparison with other studies where these temporal parameters of gait were measured by means of other methodologies; and to the applicability of the low-cost footswitch system to different types of footwear. 
(a)

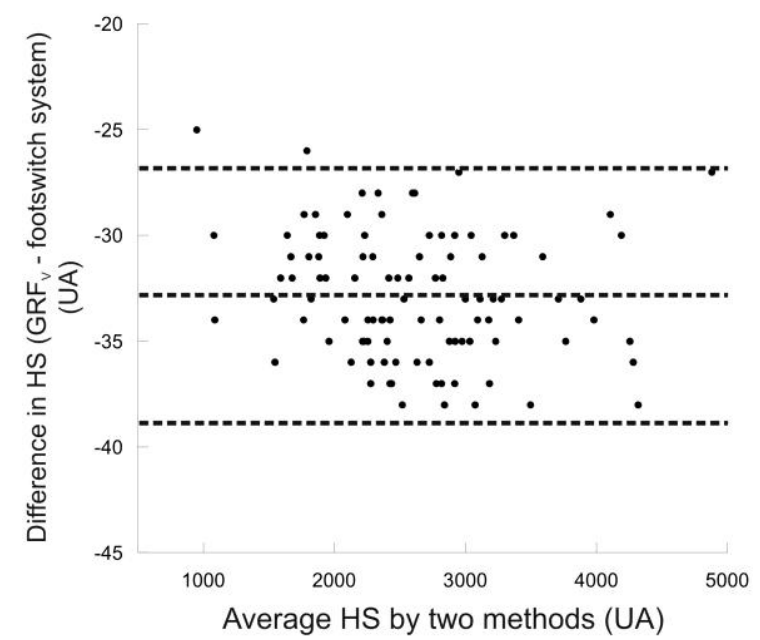

(b)

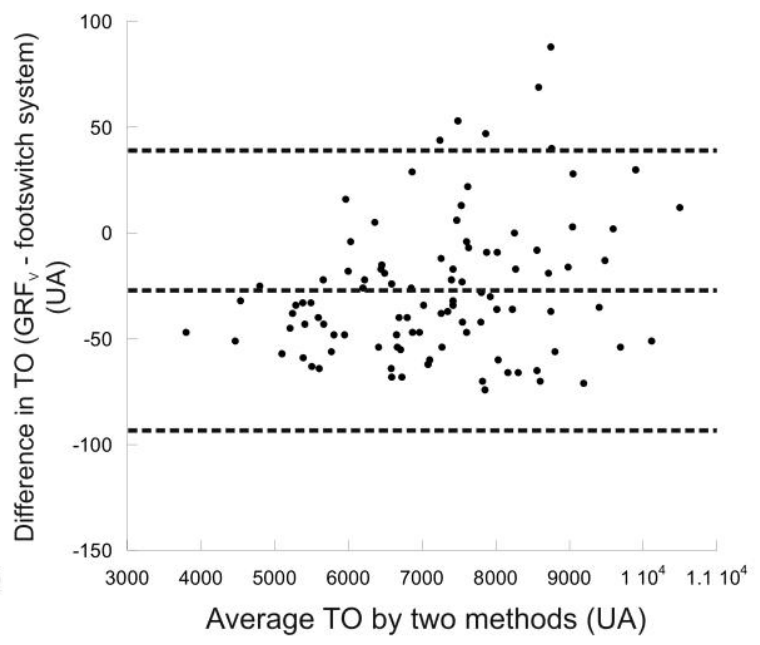

Fig. 3: Bland and Altman's limits of agreement analysis plot for (a) heel strike (HS; $98 \%$ of plots between limits of agreement) and (b) toe off (TO; 95\% of plots between limits of agreement). The abscissa axis is the force plate and footswitch system instant frame detection of HS and TO (i.e., mean frame of the two methods for each trial) and, in the ordinate axis, the difference between the two methods is assessed.

(a)

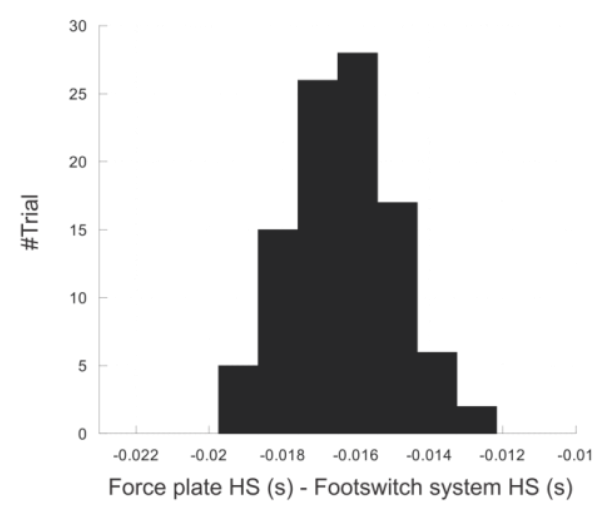

(b)

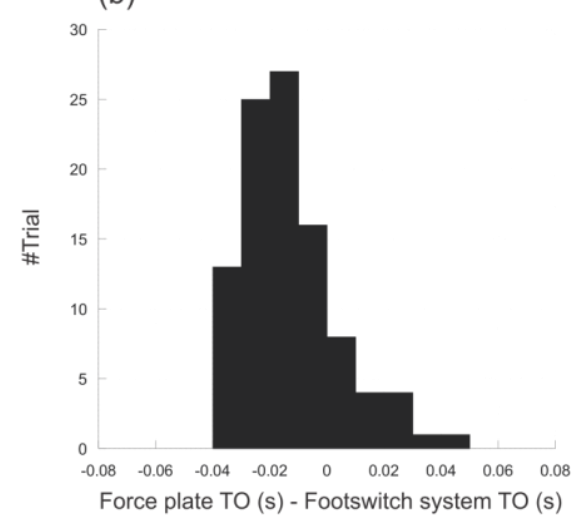

Fig. 4: Histograms describing differences between footswitch and force plate estimates of heel strike (HS) and toe off (TO) for all 100 trials. The ordinate axis is the trial number (0-100) and the abscissas axis is the difference between methods (i.e., force plate timing - footswitch system timing). These histograms demonstrate the statistical distribution of the footswitch system and force plate timing residual. For HS, the difference ranged from $20 \mathrm{~ms}$ to $-12 \mathrm{~ms}$, and for TO, the difference ranged from $-40 \mathrm{~ms}$ to $50 \mathrm{~ms}$.

Table 1: Mean, SD and CoV for the temporal parameters measurements by means of the footswitch during treadmill walking at $4.5 \mathrm{~km} \cdot \mathrm{h}^{-1}$ with two different types of footwear.

\begin{tabular}{ccccc}
\hline & \multicolumn{2}{c}{ Athletic shoe } & \multicolumn{2}{c}{ Instability shoe } \\
\hline & Mean \pm SD & CoV \pm SD & Mean \pm SD & CoV \pm SD \\
Contact time (s) & $0.752 \pm 0.033$ & $1.52 \pm 0.61$ & $0.744 \pm 0.029$ & $1.90 \pm 0.73$ \\
Balance time (s) & $0.392 \pm 0.036$ & $2.17 \pm 0.95$ & $0.401 \pm 0.023$ & $2.57 \pm 0.95$ \\
Stride time (s) & $1.144 \pm 0.053$ & $0.84 \pm 0.28$ & $1.145 \pm 0.042$ & $1.12 \pm 0.53$ \\
\hline
\end{tabular}

\section{Discussion}

Various sensors are capable of analyzing gait, ranging from simple force based switches to complex setups involving multiple inertial sensors and advanced algorithms. However additional effort is needed to minimize donning/doffing efforts and to overcome cosmetical aspects [19]. Furthermore, economical and practical issues demand a simple and inexpensive alternative for this function [8]. The present study describes the construction and validation of a low-cost footswitch system $(<10$ USD) with no voltage source or amplification needed, thus requiring a smaller number of wires and offering a low cost solution to developing biomechanics laboratories. 
Comparison between this footswitch system and the gold standard showed differences of $(16 \pm 1) \mathrm{ms}$ and $(20 \pm 9) \mathrm{ms}$ for HS and TO, respectively, and a good relation with the Bland and Altman's limits of agreement. These results are similar to the results of Hausdorff et al., [6] who described a low-cost resistive footswitch system with differences of $10 \mathrm{~ms} \pm 3$ $\mathrm{ms}$ and $22 \mathrm{~ms} \pm 8 \mathrm{~ms}$ for $\mathrm{HS}$ and TO, respectively. In addition to the good agreement with previous studies the piezoelectric footswitch system developed in the present study, as a passive sensor instrumented insole, has the main advantage, when compared to Hausdorff et al., [6] footswitch system, to be non-power supplied - thus a smaller number of cables has to be attached to the footswitch system.

Temporal parameters $\mathrm{CoV}$ (Table 1) showed good agreement with normal gait values found in the literature $(2.6 \% \pm 0.8 \%$ for contact time, $3.8 \% \pm 1.1 \%$ for balance time and $1.6 \pm 0,6 \%$ for stride time; acquisition through pressure sensor GAITRite ${ }^{\circledR}$ System - Gold GAITRite; CIR Systems, USA) [18]. In the present study, for the instrumented athletic and instability shoe respectively, CoVs of $(1.52 \pm 0.61) \%$ and $(1.90 \pm 0.73) \%$ for contact time, $(2.17 \pm 095) \%$ and $(2.57 \pm 0.95) \%$ for balance time, $(0.84 \pm 0.28) \%$ and $(1.12 \pm 0.53) \%$ for stride time were shown. The good agreement of both instrumented footwear $\mathrm{CoV}$ with literature data shows a good applicability of the footswitch system to different types of footwear; thus footwear type is not an intervening variable for the footswitch system developed in the present study.

Utilization of a footswitch system inside the footwear can lead to a delay up to 40ms in the detection of HS when compared to the force plate [7]. There are studies in the literature that used footswitch systems mounted on barefoot subjects directly to the foot sole [3,9]. Therefore, footswitch system adaptation to operate in barefoot walking may reduce the response delay when compared to the gold standard. Furthermore, possible adjustments in the construction and data processing of the present footswitch system can minimize the artifacts from the footswitch system signal, making our results closer to the results from the gold standard.

\section{Conclusion}

The present study described the entire process of construction and validation of a low-cost footswitch system with good agreement between the two different methods (i.e., force plate and footswitch system). To the authors knowledge it was the first work to fully describe and validate a piezoelectric low-cost footswitch system and it has an important practical applicability, mostly for emerging and developing countries biomechanics labs.

\section{References}

[1] R. Enoka, Bases Neuromecânicas da Cinesiologia, Manole, São Paulo, Brazil, 2000.

[2] D.A. Winter, Biomechanics and motor control of human movement, John Wiley \& Sons Ltda, New Jersey, USA, 2005.

[3] J.K. Lee, E.J. Park, Quasi real-time gait event detection using shank-attached gyroscopes, Med Biol Eng Comput. 49(6) (2011) 707-712.

[4] J. Perry, Gait analysis: normal and pathological function, Thorofare, New Jersey, USA, 1992.

[5] T. Andriacchi, E. Alexander, Studies of human locomotion: past, present and future, J Biomech. 33(10) (2000) $1217-1224$.

[6] J. Hausdorff, Z. Ladin, J. Wei, Footswitch system for measurement of the temporal parameters of gait, J Biomech. 28(3) (1995) 347-351.

[7] J. Wall, J. Crosbieb, Accuracy and reliability of temporal gait measurement, Gait \& Posture 4(4) (1996) $293-296$.

[8] P. Araújo, R. Kirkwood, E. Figueiredo, Validity and intra- and inter-rater reliability of the Observational Gait Scale for children with spastic cerebral palsy, Rev Bras Fisioter. 13(3) (2009) 267-273.

[9] J. Brinkmann, J. Perry, Rate and range of knee motion during ambulation in healthy and arthritic subjects, Phys Therapy. 65(7) (1985) 10551060 .

[10] C. Powers, L. Boyd, L. Torburn, J. Perry, Stair ambulation in persons with transtibial amputation: analysis the Seattle LightFoot, J Rehab Res Development. 34(1) (1997) 9-18.

[11] A. Mansfield, G. Lyons, The use of accelerometry to detect heel contact events for use as a sensor in FES assisted walking, Med Eng \& Physics. 25 (2003) 879-885.

[12] T. Kesar, R. Perumal, D. Reisman, et al., Functional electrical stimulation of ankle plantarflexor and dorsiflexor muscles, Stroke 40(12) (2009) 3821-3827.

[13] M. Hanlon, R. Anderson, Real-time gait event detection using wearable sensors, Gait \& Posture 30(4) (2009) $523-527$.

[14] B. Auvinet, G. Berrut, C. Touzard, et al., Reference data for normal subjects obtained with an accelerometric device, Gait \& Posture 16(2) (2002) 124-134.

[15] J. Lee, S. Cho, Y. Lee, H. Yang, J. Lee, Portable activity monitoring system for temporal parameters of gait cycles, J Med Syst. 34(5) (2010) 959-966.

[16] S. Bus, A. Lange, A comparison of the 1-step, 2-step, and 3-step protocols for obtaining barefoot plantar pressure data in the diabetic neuropathic foot, Clinical Biomech. 20(9) (2005) 892-899.

[17] J. Bland, D. Altman, Statistical methods for assessing agreement between two methods of clinical measurement, Lancet. 327(8476) (1986) 307-310.

[18] O. Beauchet, G. Allali, C. Annweiler, et al., Gait variability among healthy adults: low and high stride-to-stride variability are both a reflection of gait stability, Gerontology 55(6) (2009) 702-706.

[19] J. Rueterbories, E. Spaich, B. Larsen, O. Andersen, Methods for gait event detection and analysis in ambulatory systems, Med Eng Phys. 32(6) (2010) 545-552. 


\section{Supplementary material}

Piezo element especifications

\section{Material}

Configuration

Metal material

Resonant frequency $(\mathrm{kHz})$

Resonant impedance $(\Omega)$

Capacitance at $1000 \mathrm{~Hz}$

Operating temperature $\left({ }^{\circ} \mathrm{C}\right)$
Piezoelectric

Plane

Brass

$2,3 \pm 0,5$

450

$38000 \pm 30 \%$

-20 to +70
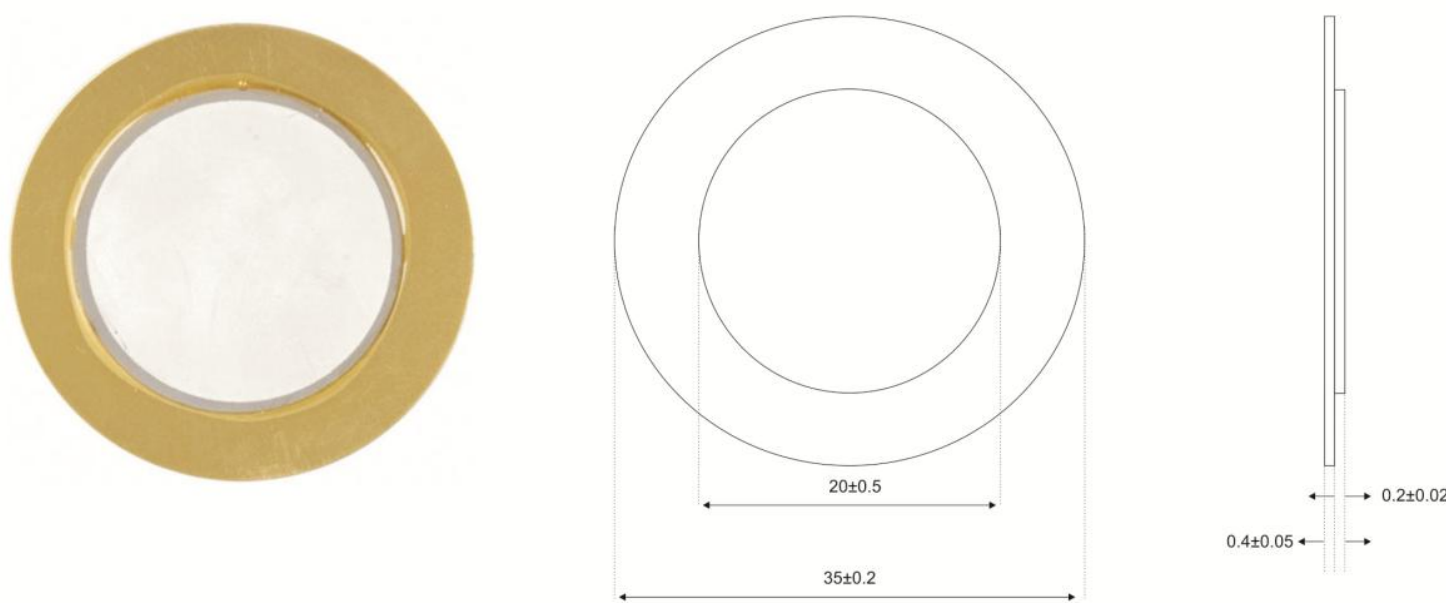